\title{
Agendas noticiosas, circularidad de saberes, riesgos y desastres ambientales: una apuesta transdisciplinaria
}

Los medios de comunicación proponen permanentemente agendas y marcos de interpretación a los públicos, a la vez que reflejan su contexto sociocultural. Esto se observa en los medios tradicionales $\mathrm{y}$ en los entornos comunicativos emergentes. Cada vez, los medios intentan mostrar de manera más notoria la participación de sus usuarios, en especial con sus huellas digitales, e incluso invitan a la co-producción y la co-creación (Scolari, 2009; Bonilla, Cataño, Rincón, \& Zuluaga, 2012). Es decir, la relación medios de comunicación y públicos siempre ha sido intrincada, y esto, progresivamente, cobra más fuerza; allí las normativas estatales e internacionales tienen un rol esencial, si bien suelen estar a la zaga de las derivas de dicha relación (Castells, 2009). Es claro, pues, que hay una enorme responsabilidad por parte de los medios en la construcción de las agendas; responsabilidad que también les atañe a las autoridades que los regulan y a los ciudadanos que los usan.

Uno de los macro-objetos de estudio importantes del campo de los estudios de ciencia, tecnología y sociedad (en adelante CTS) tiene que ver, justamente, con la manera como circulan los saberes entre medios de comunicación -en particular los noticiosos-, autoridades, científicos y expertos, grupos de interés y ciudadanía en general. En este sentido, el sub-campo de la comunicación pública de la 
ciencia y la tecnología (en adelante CPCyT) tiene mucho que aportarle y tiene retos permanentes desde y con los estudios de CTS; un sub-campo que, también, se alimenta teórica y metodológicamente del campo de los estudios de la comunicación (en adelante EC) -que asimismo tiene mucho que aprender de las perspectivas CTS-. Sin embargo, en América Latina, y especialmente en Colombia, falta mucho por avanzar en las conexiones entre los campos de los estudios CTS y de los EC, pasando por los desarrollos de la CPCyT (Cortassa, 2014; Massarani et al, 2017; Hermelin, 2018); una deuda que se extiende a un ámbito internacional más amplio (Bucchi y Trench, 2014; Kreimer, 2015). En suma, para los estudios de CTS es esencial ocuparse de los vínculos entre la circulación de saberes y las agendas de los diferentes actores, en especial las de los medios, para lo que hay que proponer ópticas de análisis en diálogo con otros campos.

Ha habido avances teóricos significativos en América Latina en los estudios de CTS como lo atestigua, por ejemplo, una buena parte de los artículos publicados por la presente revista, Trilogía, desde 2009; no obstante, hay tareas pendientes en su correspondiente campo de prácticas, sobre todo en lo referente a la construcción de informaciones noticiosas. Existen vacíos importantes en el tratamiento de los temas de ciencia y tecnología en los medios informativos latinoamericanos de mayor consumo; vacíos particularmente notorios en lo concerniente a que los debates sobre CTS permeen las agendas mediáticas como se desprende e.g. de Massarani y Ramalho (2012). Asuntos ligados a riesgos individuales y colectivos como los relacionados con el medio ambiente señalan obstáculos y desafíos en esta dirección. En un tema de tan alto impacto como el cambio climático hay estudios que muestran que el contraste entre fuentes es escaso, y se observan carencias en las discusiones entre múltiples actores, como se deriva e.g. de Arcila-Calderón, Mercado, Piñuel-Raigada, \& Suárez-Sucre (2015) para el contexto mediático hispanoparlante, y de Lema-Velez, Hermelin, Fontecha, \& Urrego (2017) para el contexto mediático colombiano.

En adelante nos centraremos en algunos retos y oportunidades que se abren, en el ámbito colombiano, para la relación entre los estudios CTS -como campo teórico, pero también como campo de prácticas-, los temas ambientales, especialmente los que tienen que ver con riesgos de desastre y las agendas mediáticas. Es menester agregar que, algunos elementos de esta discusión pueden extrapolarse a otros ámbitos. Primero, hay que decir que Colombia, a tono con el contexto latinoamericano e internacional, ha avanzado en el campo de la gestión del 
riesgo de desastres (GRD en adelante) en las últimas décadas. Esto tras padecer una inmensa cantidad de desastres de grandes proporciones y de graves consecuencias, pero también de sumatorias de pequeños desastres, que dan como resultado un número ingente de víctimas y de daños, anualmente (Campos et al, 2012). Una buena parte de la población colombiana está expuesta a amenazas, en grado alto o medio, de sismos, de inundaciones y de movimientos de masa, entre otros tipos de amenaza (UNGRD, 2014). Dicho sea de paso, en los avances en GRD ha habido injerencia de los cubrimientos noticiosos, aunque también se han conseguido a pesar de las grandes falencias de estos últimos. Todo esto ha dado lugar a normativas coyunturales, como la Ley 1523 de 2012 (Congreso de la República, 2012), que dan pautas para una estricta regulación en el manejo del riesgo de desastres en los niveles nacional, regional y local. En las políticas de GRD se ha visto una acentuación del foco en los riesgos de desastres como construcción social, como algo inherente al desarrollo de las relaciones entre las comunidades y el territorio; esto invita a un cambio de paradigma que deja atrás la prevalencia de concebir los desastres, e incluso sus riesgos, como algo inevitable (García Acosta, 2005; Lavell, 2006; Gellert-de-Pinto, 2012). Por otro lado, como lo sugiere Cortés Fonnegra (2018), se abren muchas posibilidades para que el campo de la GRD se nutra de las perspectivas CTS y viceversa, particularmente en el contexto colombiano. Verbigracia, dado que las políticas de GRD están interrelacionadas con políticas científicas que, a su vez, son tipos de políticas públicas, y responden a intereses de diversos actores de los sistemas nacionales e internacionales de ciencia y tecnología (Escobar Ortiz, 2018), las aproximaciones desde los estudios CTS son ineludibles, más aún en un país como Colombia.

Segundo, debe anotarse que, teniendo en cuenta lo anterior y dado lo que se planteó previamente la necesidad de cruces entre los estudios CTS y los EC -pasando por el sub-campo de la CPCyT-, hay que sumar a dichos cruces el campo de la GRD en el contexto académico colombiano (e incluso en el latinoamericano y en el internacional). La circulación de saberes en la que intervienen los medios de comunicación y sus agendas en relación con los problemas ambientales, los desastres y sus riesgos, apuntan hacia la relevancia de estas apuestas inter-campos en un país como Colombia. Un objeto de estudio de la naturaleza del de dicha circulación invita a abordajes teóricos y metodológicos múltiples. Veamos lo que arrojan estos trabajos: en Daza-Caicedo y Lozano-Borda (2014) se muestra cómo, en Colombia, la ciudadanía les da preponderancia a los medios de comunicación 
para acercarse a problemas y saberes de carácter tecnocientífico. En Daza-Caicedo (2009) se observa la misma tendencia, más marcada aún en lo que respecta a los temas ambientales; cuando se trata de riesgos de esta índole, las personas se consideran bastante interesadas e incluso apropiadas o conocedoras de tales saberes. Por su parte, en Barrios, Arroyave, \& Vega (2017) y en Obregón, Arroyave, $\&$ Barrios (2009) se muestra la dificultad de medios noticiosos latinoamericanos de alto impacto -entre ellos, colombianos- para dar cuenta del cambio de paradigma frente a la GRD, antes mencionado. Allí se observa también la relativa poca presencia de científicos y expertos como fuentes para estos temas. Así, en lo concerniente a la concepción de riesgos ambientales y desastres, hay indicios de que existen cortocircuitos entre las agendas demandadas por los ciudadanos y las agendas que proponen los medios noticiosos en un contexto como el colombiano, a lo que se suman algunas dificultades para que los saberes tecnocientíficos sean consuetudinarios en ambas agendas.

Ahora bien, con lo que se acaba de exponer no se intenta defender que en la circularidad de saberes entre diversos actores, los saberes tecnocientíficos deban ser los siempre predominantes en aras de fortalecer la GRD. La circularidad invita, precisamente, a que se les dé importancia a los repertorios culturales de los diferentes actores, incluidas las múltiples racionalidades, allende la racionalidad ilustrada como "una razón única y valedera"; múltiples racionalidades que se requieren para comprender las complejas relaciones sociales y mediáticas con los riesgos en un contexto como el colombiano, siguiendo a Márquez (2008). Esto nos incita a pensar más en términos de modelos democráticos que de modelos deficitarios de CPCyT (Lozano, 2005; Rivera Berrío, 2010; Pérez-Bustos y LozanoBorda, 2011), es decir, modelos horizontales y no verticales entre los supuestos ilustrados y los supuestos legos. (Cabe subrayar que, como se deriva de Lundgren y MacMakin (2009), en el sub-campo de la comunicación del riesgo, sus modelos más prescriptivos son muy próximos a los modelos deficitarios; en contraste, sus modelos más dialógicos son muy próximos a los modelos democráticos). Algo que implica entender los gradientes de saber entre distintos actores como condiciones de posibilidad para la actividad y la co-construcción de conocimientos, según lo aduce Rancière (2010). Evidentemente los medios noticiosos tienen en todo esto un rol fundamental: no son un actor cualquiera en la circularidad en virtud de que constituyen vasos comunicantes por antonomasia entre una variedad de actores, y son los llamados a hacer contrapesos frente a otros poderes; en ambos sentidos 
contribuyen al tejido social ligado a la concepción de los riesgos ambientales (De Cheveigné, 2000), específicamente a la GRD. Dichos medios están llamados a usar modelos democráticos, con una miscelánea de voces, que favorezcan el debate a fin de empoderar los diferentes segmentos sociales, en lugar de ceñirse a hacer simples traducciones de las posturas y los lenguajes de autoridades epistémicas y estatales. Sin embargo, los medios noticiosos, a su vez, deben ponderar cuándo se hace inexorable usar modelos deficitarios, al menos parcialmente, a la hora de hacerle frente a una situación de crisis como sucede con un desastre inminente. Por supuesto, estudiar funciones mediáticas como las que acabamos de mencionar es una de las tareas para lo que hemos denominado aquí apuestas inter-campos.

Para abordar problemáticas como las que hemos expuesto es necesario entender los contextos socioculturales, históricos y políticos en los que se constituyen e interactúan las agendas de los diferentes actores, entre ellas, claro está, las de los medios noticiosos. En nuestro caso es preciso destacar particularidades del pasado reciente y del presente de un país como Colombia. Una esencial y que se ha reflejado en las agendas noticiosas en los últimos años tiene que ver con el conflicto armado. Tras las negociaciones con los grupos paramilitares del gobierno nacional del periodo de 2002 a 2010 y tras las negociaciones y la firma de los acuerdos de paz con la guerrilla de las FARC del gobierno nacional del periodo de 2010 a 2018, las prioridades del acontecer noticioso han cambiado significativamente. Las cifras de los gobiernos y las cifras de otros entes independientes han mostrado una disminución sustancial de los homicidios, las masacres y los secuestros. De hecho, se habla de que en 2017 se llegó a la tasa de homicidios más baja en 42 años (El espectador, 2018). Además, el conflicto armado no ha copado solo las agendas de los medios noticiosos, de las autoridades, y la ciudadanía: ha prevalecido en buena parte de los análisis de medios de los académicos en Colombia (Tamayo y Bonilla, 2014). Estas variaciones en los indicadores de violencia se reflejan en que otros temas han cobrado o pueden cobrar importancia. De hecho, se trata de una oportunidad para que los diferentes actores les den más cabida a otras temáticas, en especial las que aquí nos ocupan: los desastres ambientales y sus riesgos. Una oportunidad que le incumbe a los medios noticiosos, a las autoridades estatales, a la academia y a la ciudadanía. Por ejemplo, un desafío que reviste relevancia consiste en la posibilidad de que los medios noticiosos centren su lupa no solo en los desastres ambientales sino en los riesgos, como consecuencia de la disminución hechos violentos ligados al conflicto armado - ¿lo que les da menos pretextos de 
esta naturaleza? -. Se trata de un reto que también les compete a los demás actores mencionados; uno que tiene que ver con que transitemos hacia una sociedad del riesgo, con sus grandes beneficios, sin perder de vista sus adversidades, siguiendo a Beck (2006).

Se ven pues las posibilidades de abonar el terreno para robustecer el campo de la GRD en Colombia, en diálogo con el de los estudios de CTS y el de los EC, en los tres casos como campos estudio, pero también con sus correspondientes campos de prácticas. En términos generales, se han dado aquí algunas puntadas sobre la necesidad de hacer apuestas inter-campos en contextos concretos. Consideramos que esta perspectiva puede ser útil en Colombia y en otras latitudes, y puede servir como una suerte de sugerencia para pensar los diversos trabajos que se publican en este número de Trilogía, una revista que, a nuestro juicio, aboga de forma decidida por la transdisciplinariedad.

\section{Daniel Hermelin}

dhermeli@eafit.edu.co Candidato a Doctor en Ciencias Humanas y Sociales Universidad Nacional de Colombia-Sede Medellín Profesor del Departamento de Comunicación Social Escuela de Humanidades Universidad EAFIT 


\section{REFERENCIAS}

Arcila-Calderón, C., Mercado, M. T., Piñuel-Raigada, J. L., \& Suárez-Sucre, E. (2015). Media coverage of climate change in Spanish-Speaking online media. Convergencia, 22(68), 71-95.

Barrios, M. M., Arroyave, J., \& Vega, L. (2017) El cambio de paradigma en la cobertura informativa de la gestión de riesgo de desastres: retos y oportunidades.Chasqui. Revista Latinoamericana de Comunicación (136), 129-144.

Beck, U. (2006). La sociedad del riesgo. Hacia una nueva modernidad. Barcelona: Paidós.

Bonilla, J. I., Cataño, M., Rincón, O., \& Zuluaga, J. (2012). De las audiencias contemplativas a los productores conectados. Cali, Medellín y Bogotá: Pontificia Universidad Javeriana, Universidad EAFIT y Universidad de los Andes.

Bucchi, M., \& Trench, B. (Eds.). (2014). Handbook of Public Communication of Science and Technology. Londres: Routledge.

Campos, A., Holm-Nielsen, N., Díaz C., Rubiano, D. M., Costa. C. R., Ramírez, F., \& Dickson, E. (Eds.). (2012). Análisis de la gestión del riesgo de desastres en Colombia: un aporte para la construcción de políticas públicas. Bogotá: Banco Mundial, GFDRR.

Castells, M. (2009). Comunicación y poder. Madrid: Alianza Editorial.

Congreso de la República. (2012). Ley 1523 de 2012. Por la cual se adopta la política nacional de gestión del riesgo de desastres y se establece el Sistema Nacional de Gestión del Riesgo de Desastres y se dictan otras disposiciones. Bogotá: Congreso de la República de Colombia. Recuperado de http:/ / www. secretariasenado.gov.co/senado/basedoc/ley_1523_2012.html

Cortassa, C. (2014). Ciencia y audiencias: aportes para consolidar una agenda de investigación. Diálogos de la Comunicación-FELAFACS, 88, 1-17.

Cortés Fonnegra, L. M. (2018). Reflexión sobre la gestión de riesgos de desastres bajo la perspectiva CTS. Trilogía Ciencia Tecnología Sociedad, 10(19), 7-10.

Daza-Caicedo, S. (Ed.). (2009). Percepciones sobre la ciencia y la tecnología en Bogotá. Bogotá: Observatorio Colombiano de Ciencia y Tecnología. 
EDITORIAL

Daza-Caicedo, S., \& Lozano-Borda, M. (Eds.). (2014). Percepciones de las Ciencias y las Tecnologías en Colombia. Resultados de la III Encuesta Nacional de Percepción Pública de la Ciencia y la Tecnología. Bogotá: Observatorio Colombiano de Ciencia y Tecnología.

De Cheveigné, S. (2000). L'environnement dans les journaux télévisés. Médiateurs et visions du monde. París: CNRS Éditions.

El Espectador. (21 de enero de 2018). Homicidios en Colombia: la tasa más baja en los últimos 42 años se dio en 2017. El Espectador. Recuperado de https: / / www.elespectador.com/noticias/judicial/homicidios-en-colombia-la-tasamas-baja-en-los-ultimos-42-anos-se-dio-en-2017-articulo-734526.

Escobar Ortiz, J. M. (2018). El diseño de la política científica en América Latina: organismos internacionales, gobiernos nacionales y comunidades científicas. Trilogía Ciencia Tecnología y Sociedad, 10(18), 7-11.

García Acosta, V. (2005). El riesgo como construcción social y la construcción social de riesgos. Desacatos. Revista de Antropología Social, 19, 11-24.

Gellert-de-Pinto, G. I. (2012). El cambio de paradigma: de la atención de desastres a la gestión del riesgo. Boletín Científico Sapiens Research, 2(1), 13-17.

Hermelin, D. (2018). Comunicación de la ciencia. Un aprendizaje desde y con las ciencias sociales y humanas. InMediaciones de la Comunicación, 13(2), 51-70.

Kreimer, P. (2015). Public understanding of science and social studies of science: convergence or parallel paths?. En B. Schiele, J. Le Marec, y P. Baranger (eds.) Science Communication Today (pp. 1-16). Nancy: PUN.

Lavell, A. M. (2006). Apuntes para una reflexión institucional en países de la Subregión andina sobre el enfoque de la Gestión del Riesgo. Lima: PREDECAN. Recuperado de http: / / www.comunidadandina.org/predecan/doc/r1/docAllan2.pdf

Lema-Velez, L. F., Hermelin, D., Fontecha, M. M., \& Urrego, D. H. (2017). Climate Change Communication in Colombia. Climate change communication. Climate science. Oxford Research Encyclopedia. Recuperado de http://climatescience. oxfordre.com / view / 10.1093 / acrefore / 9780190228620.001 .0001 / acrefore9780190228620-e-598

Lozano, M. (2005). Programas y experiencias en popularización de la ciencia y la tecnología. Panorámica desde los países del Convenio Andrés Bello. Bogotá: Convenio Andrés Bello. 
Lundgren, R. E., \& MacMakin, A. H. (2009). Risk communication. A handbook for communicating environmental, safety, and health risks. New Jersey: IEE Press y John Wiley \& Sons.

Márquez, J. (2008). Ciencia, riesgos colectivos y prensa escrita. El caso del sida en Colombia. Medellín: Universidad Nacional de Colombia.

Massarani, L. et al. (2017). Aproximaciones a la investigación en divulgación de la ciencia en América Latina a partir de sus artículos académicos. Río de Janeiro, Brasil: Fiocruz.

Massarani, L., \& Ramalho, M. (Eds.). (2012). Monitoramento e capacitação em jornalismo científico - a experiência de uma rede ibero-americana. Rio de Janeiro y Quito: Museu da Vida/Casa de Oswaldo Cruz y Ciespal.

Obregón, R., Arroyave, J., \& Barrios, M. M. (2009). Cubrimiento periodístico de la gestión del riesgo en la Subregión Andina: discursos periodísticos y perspectivas desde la comunicación para el cambio social. Lima: PREDECAN, Comunidad Andina de Naciones.

Pérez-Bustos, T., \& Lozano-Borda, M. (Eds.). (2011). Ciencia, tecnología y democracia. Reflexiones en torno a la apropiación social del conocimiento. Medellín: Colciencias y Universidad EAFIT.

Rancière, J. (2010). El espectador emancipado. Buenos Aires: Manantial.

Rivera Berrío, J. G. (2010). La comunicación del riesgo: hacia un modelo efectivo y situacional. Medellín: Fondo Editorial ITM.

Scolari, C. (2009). Alrededor de la(s) convergencia(s). Conversaciones teóricas, divergencias conceptuales y transformaciones en el ecosistema de medios. Signo y Pensamiento, 28(54), 44-55.

Tamayo, C., \& Bonilla, J. I. (2014). El deber de la memoria. La agenda investigativa sobre la cobertura informativa del conflicto armado en Colombia, 2002-2012. Palabra Clave, 17(1), 13-45.

Unidad Nacional para la Gestión del Riesgo de Desastres (UNGRD). (2014). Documento de priorización de lineas estratégicas y zonas de intervención en gestión del riesgo de desastres en Colombia (2014-2018). Bogotá: UNGRD. 